\title{
Lipid exposure elicits differential responses in gene expression and DNA methylation in primary human skeletal muscle cells from severely obese
}

\section{women}

\author{
Jill M. Maples, ${ }^{1,2}$ (D) Jeffrey J. Brault, ${ }^{3,4,5,6,7}$ Brian M. Shewchuk, ${ }^{6}$ (D) Carol A. Witczak, ${ }^{3,4,5,6,7}$ Kai Zou, $^{3,4,5}$ \\ Naomi Rowland, ${ }^{2}$ Monica J. Hubal, ${ }^{8}$ Todd M. Weber, ${ }^{3,4,5}$ and Joseph A. Houmard ${ }^{3,4,5}$ \\ ${ }^{1}$ School of Kinesiology, Recreation, and Sport, Western Kentucky University, Bowling Green, Kentucky; ${ }^{2}$ Biotechnology \\ Center, Western Kentucky University, Bowling Green, Kentucky; ${ }^{3}$ Human Performance Laboratory, East Carolina University, \\ Greenville, North Carolina; ${ }^{4}$ Department of Kinesiology, East Carolina University, Greenville, North Carolina; ${ }^{5}$ East \\ Carolina Diabetes and Obesity Institute, East Carolina University, Greenville, North Carolina; ${ }^{6}$ Department of Biochemistry \\ \& Molecular Biology, East Carolina University, Greenville, North Carolina; ${ }^{7}$ Department of Physiology, East Carolina \\ University, Greenville, North Carolina; and ${ }^{8}$ Department of Integrative Systems Biology, Children's National Medical Center, \\ Washington, District of Columbia
}

Submitted 22 May 2014; accepted in final form 4 February 2015

\begin{abstract}
Maples JM, Brault JJ, Shewchuk BM, Witczak CA, Zou K, Rowland N, Hubal MJ, Weber TM, Houmard JA. Lipid exposure elicits differential responses in gene expression and DNA methylation in primary human skeletal muscle cells from severely obese women. Physiol Genomics 47: 139-146, 2015. First published February 10, 2015; doi:10.1152/physiolgenomics.00065.2014.- The skeletal muscle of obese individuals exhibits an impaired ability to increase the expression of genes linked with fatty acid oxidation (FAO) upon lipid exposure. The present study determined if this response could be attributed to differential DNA methylation signatures. RNA and DNA were isolated from primary human skeletal muscle cells (HSkMC) from lean and severely obese women following lipid incubation. mRNA expression and DNA methylation were quantified for genes that globally regulate FAO [PPAR $\gamma$ coactivator $(P G C-1 \alpha)$, peroxisome proliferator-activated receptors (PPARs), nuclear respiratory factors $(N R F s)]$. With lipid oversupply, increases in $N R F-1, N R F-2$, $P P A R \alpha$, and PPAR $\delta$ expression were dampened in skeletal muscle from severely obese compared with lean women. The expression of genes downstream of the PPARs and NRFs also exhibited a pattern of not increasing as robustly upon lipid exposure with obesity. Increases in $\mathrm{CpG}$ methylation near the transcription start site with lipid oversupply were positively related to $P P A R \delta$ expression; increases in methylation with lipid were depressed in HSkMC from severely obese women. With severe obesity, there is an impaired ability to upregulate global transcriptional regulators of FAO in response to lipid exposure. Transient changes in DNA methylation patterns and differences in the methylation signature with severe obesity may play a role in the transcriptional regulation of $P P A R \delta$ in response to lipid. The persistence of differential responses to lipid in HSkMC derived from lean and obese subjects supports the possibility of stable epigenetic programming of skeletal muscle cells by the respective environments.
\end{abstract}

obesity; DNA methylation; skeletal muscle

METABOLIC FLEXIBILITY, THE ability to adjust substrate oxidation according to nutrient availability, is a critical aspect of metabolic health and is compromised with obesity (17). Indicative of metabolic flexibility, a high-fat diet increases fatty acid oxidation (FAO) and the expression of genes involved with

Address for reprint requests and other correspondence: J. A. Houmard, Dept. of Kinesiology, Human Performance Laboratory, East Carolina Univ., 380 Ward Sports Medicine Bldg., Greenville, NC 27858 (e-mail: houmardj@ecu. edu).
FAO [i.e., peroxisome proliferator-activated receptor $(P P A R) \alpha$ and PPAR $\gamma$ coactivator- $1 \alpha(P G C-1 \alpha)]$ in skeletal muscle from lean individuals $(7,8,10,11,13,14)$. To the contrary, this adjustment to lipid is largely absent in skeletal muscle from severely obese subjects (7), which in turn can predispose these individuals to ectopic lipid accumulation and metabolic disease. Despite such potentially detrimental health outcomes, the regulatory mechanisms that differentially control gene expression and thus possibly limit metabolic flexibility in the skeletal muscle of severely obese individuals in response to lipid are not defined.

Acute epigenetic modifications of the genome (e.g., DNA methylation) may provide a connection between nutritional intake and the regulation of gene expression. Studies have shown transient alterations in DNA methylation in human skeletal muscle with either high-fat feedings or lipid incubations in primary human skeletal muscle cell (HSkMC) cultures $(3,16)$. However, no studies have examined DNA methylation signatures as a possible mechanism for the differential responses of gene expression to lipid in the skeletal muscle of obese vs. lean individuals. In addition, the extent to which stable epigenetic changes apart from continued systemic stimuli contribute to differences in skeletal muscle metabolism between lean and obese individuals is unclear. This study tested the hypothesis that DNA methylation patterns for key genes critical in FAO would differ in response to lipid exposure in human skeletal muscle with severe obesity and in turn be related to gene expression. We utilized the HSkMC model to test this hypothesis as: 1) the phenotype in terms of a reduction in FAO with obesity is retained (15), and 2) we can study the molecular adaptations to a lipid stimulus in an environment void of in vivo hormonal and neural stimuli and thus intrinsic to skeletal muscle itself, addressing the potential for epigenetic programming of cell function in the lean and obese environments.

\section{MATERIALS AND METHODS}

Study design. The study was designed to compare the responses of genes linked with FAO to lipid oversupply in lean vs. severely obese subjects and determine if transient epigenetic modifications (DNA methylation) play a role in regulating gene expression and the differences seen with obesity. The study focused on the PPARs and nuclear 
respiratory factors (NRFs) because of their importance in activating broad gene expression programs critical to mitochondrial function and FAO $(18,19,22,23)$. PPAR- and NRF-regulated genes involved in mitochondrial function and FAO were also examined. Skeletal muscle biopsies were obtained from the vastus lateralis of lean and severely obese women and used to derive primary HSkMC cultures. After differentiation into myotubes, HSkMC were incubated in a physiologically relevant lipid mixture (30) $(250 \mu \mathrm{M}$ oleate-palmitate) for $48 \mathrm{~h}$, and mRNA expression and DNA methylation determined.

Materials. All chemical reagents/substrates were purchased from Sigma (St. Louis, MO) unless otherwise stated. Dulbecco's phosphate-buffered saline (DPBS), fetal bovine serum, heat-inactivated horse serum, gentamicin, 0.05\% trypsin EDTA, and Hanks's balanced salt solution were obtained from Invitrogen (Grand Island, NY). Growth media and differentiation media consisted of low-glucose (5 mmol/l) Dulbecco's modified Eagle's medium from Invitrogen. Type I collagen-coated tissue culture plates were obtained from Becton Dickinson (Franklin Lakes, NJ). PCR reagents were purchased from Applied Biosystems (Foster City, CA).

Human subjects. Muscle biopsies were obtained by the percutaneous needle biopsy technique (12) under local anesthesia $(0.01 \%$ lidocaine) from the vastus lateralis of nine lean [body mass index $\left.(\mathrm{BMI}) \leq 25.0 \mathrm{~kg} / \mathrm{m}^{2}\right]$ and 10 obese women approximating class III (severe) obesity ( $\geq 40 \mathrm{~kg} / \mathrm{m}^{2}$ ). Participants were Caucasian, relatively young, free from overt disease, nonsmokers, and not taking medications known to alter metabolism. All procedures were approved by the East Carolina University Institutional Review Board.

Primary HSkMC. Satellite cells were isolated from $\sim 50-100 \mathrm{mg}$ of fresh muscle and cultured as previously described (4). For experiments, cells were subcultured into T-150 flasks and $10 \mathrm{~cm}$ dishes. Upon reaching $\sim 80-90 \%$ confluence, differentiation was induced by switching the growth media to low-serum differentiation media containing $2 \%$ heat-inactivated horse serum, $0.05 \mathrm{mg} / \mathrm{ml}$ fetuin, and 5 $\mu \mathrm{g} / \mathrm{ml}$ gentamicin. On day 5 of differentiation, myotubes were given fresh differentiation media supplemented with 1) $0.1 \%$ bovine serum albumin (BSA) + $1 \mathrm{mM}$ carnitine (control) or 2) $250 \mu \mathrm{M}$ oleatepalmitate (1:1 ratio) bound to $0.1 \% \mathrm{BSA}+1 \mathrm{mM}$ carnitine (lipid) for a total incubation period of $48 \mathrm{~h}$. Myotubes were harvested on day 7 similar to previous work (24).

$m R N A$ quantification. Total RNA was isolated with the RNeasy Mini Kit (Qiagen, Valencia, CA) with on-column DNase digestion using the RNase-Free DNase Set (Qiagen) to remove residual DNA. RNA was quantified with the NanoDrop 1000 Spectrophotometer from Thermoscientific (Wilmington, DE). We determined concentration by measuring absorbance at $260 \mathrm{~nm}$, and purity was assessed with the 260:280 ratio. We reverse transcribed $2 \mu \mathrm{g}$ RNA into cDNA, and PCR was performed in triplicate with the Applied Biosystems ABI $7900 \mathrm{HT}$ sequence detection instrument and software with Taqman Universal PCR Master Mix and TaqMan gene expression assays (Applied Biosystems) in accordance with manufacturer's instructions. Reactions were run with the following thermal cycling conditions: $50^{\circ} \mathrm{C}$ for $2 \mathrm{~min}, 95^{\circ} \mathrm{C}$ for $10 \mathrm{~min}, 40$ cycles of $95^{\circ} \mathrm{C}$ for $15 \mathrm{~s}$ followed by $60^{\circ} \mathrm{C}$ for $1 \mathrm{~min}$. mRNA content was measured in triplicate by the comparative $\mathrm{Ct}$ method with a multiplexed endogenous control (18S) and converted to a linear function by a base 2 antilog transformation.

Bisulfite conversion and DNA methylation profiling. Cells were washed with DPBS and trypsinized with trypsin-EDTA (0.05\% trypsin and $0.25 \%$ EDTA). Total DNA was extracted with a QIAamp DNA mini kit (Qiagen), and total DNA quantified with the NanoDrop 1000 Spectrophotometer from Thermoscientific. We determined concentration by measuring absorbance at $260 \mathrm{~nm}$, purity was assessed with the 260:280 ratio, and all samples having a ratio $>1.8$ were used in subsequent analysis.

500 ng DNA was bisulfate converted with the EZ DNA Methylation Kit (Zymo Research, Orange, CA), with the alternative incubation conditions recommended for the Illumina Infinium Methylation Assay. Genome-wide DNA methylation analysis was conducted on bisulfate treated DNA samples using the Illumina Infinium Human Methylation 450K BeadChip, allowing the quantitative monitoring of 485,764 cytosine positions (28). We amplified and fragmented $12 \mu \mathrm{l}$ of each bisulfate-converted sample following the manufacturer's protocol, hybridized it to arrays in a balanced design, and scanned it on an Illumina iScan System. Data were analyzed with Illumina's Genome Studio software.

Western blot. Cells were washed twice with ice-cold PBS and harvested in $400 \mu \mathrm{l}$ RIPA lysis buffer [150 mM NaCl, $1 \%$ Triton $\mathrm{X}-100,0.5 \%$ sodium deoxycholate, $0.1 \%$ SDS, $50 \mathrm{mM}$ Tris ( $\mathrm{pH} 8.0$ )] supplemented with protease and phosphatase cocktail inhibitors (Roche Diagnostics). Samples were sonicated and centrifuged at $20,000 \mathrm{~g}$ for $30 \mathrm{~min}$ at $4^{\circ} \mathrm{C}$. Protein concentrations were determined from cell extracts with the bicinchoninic acid assay (Pierce Biotechnology). Cellular protein $(30 \mu \mathrm{g})$ was separated by SDS-PAGE, electrotransferred onto polyvinylidene difluoride membranes (Millipore), and probed overnight for the proteins of interest: $\operatorname{PPAR} \alpha$, PPAR $\delta$, NRF-2, pyruvate dehydrogenase kinase 4 (PDK4), and cytochrome c (CYCS) (AbCam). Membranes were incubated for $1 \mathrm{~h}$ at room temperature with the corresponding secondary antibody, and the immunoreactive proteins were detected and quantified by enhanced chemiluminescence (FluorChem HD2 System, Protein Simple). A control sample was visualized on each gel to ensure consistency, and samples were normalized for blotting efficiency to an endogenous control (Vinculin, AbCam).

$F A O$. After a $48 \mathrm{~h}$ incubation period with either control or lipid media, FAO was determined. In brief, myotubes were incubated at $37^{\circ} \mathrm{C}$ in sealed 12 -well plates containing differentiation media supplemented with $12.5 \mathrm{mM}$ HEPES, 0.5\% BSA, $1 \mathrm{mM}$ carnitine, 250 $\mu \mathrm{M}$ sodium oleate, and $1 \mu \mathrm{Ci} / \mathrm{ml}\left[1-{ }^{14} \mathrm{C}\right]$ oleate (Sigma-Aldrich) for $3 \mathrm{~h}$. After the incubation period, the medium was transferred to new plates and assayed for the collection of ${ }^{14} \mathrm{CO}_{2}$ production (complete oleate oxidation), which was quantified via liquid scintillation counting using $4 \mathrm{ml}$ of Uniscint BD (National Diagnostics, Atlanta, GA) (24). FAO was determined in four lean and four severely obese women.

Statistical analysis. Statistical analyses were performed using PASW Statistics 19 software (SPSS, Chicago, IL) on raw or logtransformed data. Comparisons between HSkMC from lean and obese donors were performed with repeated-measures ANOVA with emphasis on a "weight status" (lean, obese) $\times$ "treatment" (control, lipid-treated) interaction, indicating that lean and severely obese individuals responded differently to lipid oversupply. Post hoc comparisons were performed with contrast-contrast analyses. All data met assumptions of sphericity and homogeneity of variance. Data are presented as means $\pm \mathrm{SE}$.

\section{RESULTS}

Participant characteristics. Participant characteristics are presented in Table 1. By design, most of the obese women had a BMI classified as class III (severe) obesity $\left(\geq 40 \mathrm{~kg} / \mathrm{m}^{2}\right)$ and were heavier than the lean women $(P \leq 0.05)$. Fasting blood glucose, cholesterol, and triglyceride values did not differ between groups. However, fasting HDL values were $\sim 20 \%$ lower, while insulin and homeostatic model assessment values were higher in the severely obese subjects $(P \leq 0.05)$. Both groups consisted of relatively young women.

Gene expression. Under the control untreated condition (i.e., prelipid exposure), there were no differences between groups in baseline PPAR $\alpha, N R F-1$, and NRF-2 mRNA content; however, $P P A R \delta$ mRNA content was $\sim 21 \%$ lower $(P \leq 0.05)$ in the obese compared with lean (data not shown). In terms of mean absolute mRNA content among the subjects, there were significant $(P \leq 0.05)$ interaction effects in response to the 
Table 1. Participant characteristics

\begin{tabular}{lrr}
\hline \hline & Lean $(n=9)$ & Obese $(n=10)$ \\
\hline Age, yr & $23.4 \pm 1.5$ & $30.2 \pm 2.6$ \\
Stature, cm & $164.7 \pm 1.8$ & $165.5 \pm 2.2$ \\
Mass, kg & $62.6 \pm 1.3$ & $113.7 \pm 6.3^{*}$ \\
BMI, kg/m ${ }^{2}$ & $22.8 \pm 0.7$ & $41.3 \pm 1.5^{*}$ \\
Plasma cholesterol, mg/dl & $179 \pm 8.2$ & $171 \pm 10.1$ \\
Plasma triglycerides, mg/dl & $102 \pm 7.4$ & $121 \pm 21.0$ \\
HDL, mg/dl & $56 \pm 4.0$ & $40 \pm 3.4^{*}$ \\
Fasting glucose, mmol/l & $4.6 \pm 0.1$ & $4.9 \pm 0.4$ \\
Fasting insulin, $\mu \mathrm{U} / \mathrm{l}$ & $4.3 \pm 1.2$ & $17.3 \pm 5.0^{*}$ \\
HOMA-IR & $0.9 \pm 0.3$ & $3.8 \pm 1.1^{*}$ \\
\hline
\end{tabular}

Data are means \pm SE. BMI, body mass index; HOMA-IR, homeostatic model assessment- insulin resistance. All subjects were women. *Significant difference $(P \leq 0.05)$ between lean and severely obese groups.

48 h 1:1 oleate-palmitate lipid treatment for PPAR $\alpha, P P A R \delta$, $N R F-1$, and $N R F-2$, where mRNA content increased $37.2 \pm$ $17.3 \%(P=0.08), 35.8 \pm 16.0 \%(P=0.04), 58.2 \pm 30.2 \%$ $(P=0.04)$, and $54.2 \pm 16.4 \%(P=0.01)$, respectively, in the lipid-treated state in HSkMC from the lean women. In contrast, in HSkMC from severely obese women mean mRNA content either decreased $(P P A R \alpha,-18.4 \pm 6.5 \%, P=0.02)$ or did not change $(P P A R \delta,-15.0 \pm 11.0 \%, P=0.09 ; N R F-1,-10.8 \pm$ $12.6 \%, P=0.21 ; N R F-2,-11.2 \pm 13.7 \%, P=0.21)$ with lipid treatment. Similarly, there were significant differences $(P \leq 0.05)$ between lean and obese in the response to lipid treatment when we compared the individual relative foldchanges (lipid treated divided by baseline control for each subject) for $P P A R \alpha, P P A R \delta, N R F-1$, and $N R F-2$ (Fig. 1). There were no differences between groups in $P G C-1 \alpha, P G C$ $1 \beta$, and PPRC1 mRNA content, nor were there any significant lipid-induced changes in the expression of these genes (Fig. 1).

In an effort to determine whether the differential expression of these transcriptional regulators had a downstream effect, four PPAR-responsive genes that play roles in FAO were analyzed: angiopoietin-like 4, citrate synthase (CS), PDK4, and mitochondrial uncoupling protein 3 (UCP3). There was a significant interaction effect for PDK4 $(P=0.02)$, where mRNA content significantly increased to a greater extent in the lean compared with the obese in response to lipid. Similarly there was a significant difference in PDK4 mRNA individual fold-changes with the lipid-induced change in mRNA being significantly lower $(P=0.02)$ in HSkMC from the severely obese (20.4 \pm 3.8-fold increase) compared with the lean $(42.0 \pm 8.1$-fold increase) women (Fig. $2 A)$. Additionally, the lipid-induced individual fold-changes in $C S$ and UCP3 mRNA content in HSkMC from the obese (1.2 \pm 0.2 - and $0.9 \pm$ 0.1 -fold increase, respectively) compared with the lean $(1.6 \pm$ 0.1 and $1.2 \pm 0.1$-fold increase) exhibited a trend $(P=0.10$ and $P=0.09$ ) for being suppressed with severe obesity. Six NRF-regulated genes were analyzed: cytochrome c oxidase subunit VIc (COX6c), CYCS, mitochondrial elongation factor G 1 (GFM1), mitochondrial ribosomal protein L2 (MRPL2), mitochondrial transcription factor $\mathrm{A}(T F A M)$, and mitochondrial transcription factor B2 (TFB2M). The lipid-induced individual fold-changes in $C Y C S$ mRNA content were significantly $(P \leq 0.05)$ lower in the severely obese $(0.9 \pm$ 0.1 -fold-change) compared with the lean $(1.3 \pm 0.2$-fold increase) women (Fig. 2B).
DNA methylation. DNA methylation was determined on 485,764 genomic cytosine positions using HumanMethylation 450 BeadChips (Illumina). Within the PPAR $\delta$ gene DNA methylation was determined on 23 sites (Fig. $3 A$ and Table 2), with nine cytosines being within 1,000 base pairs (bp) of the transcription start site (TSS). For PPARS (Fig. 3, $B$ and $C$ ), there were significant increases in methylation with lipid treatment for the cytosines at positions 6 and 7 ( -71 and $-61 \mathrm{bp}$ relative to the TSS) in the lean, but not severely obese, women. As a result of lipid oversupply the methylation of the cytosine at position 6 and 7 increased by $1.3 \pm 0.12$ and $1.5 \pm 0.2$-fold, respectively, in the lean $(P \leq 0.05)$ but did not change in the severely obese women. Lipid oversupply also resulted in an increase from $59.7 \pm 4.0 \%$ to $71.8 \pm 3.9 \%(P \leq 0.05)$ in the methylation of the cytosine at position 23 in HSkMC in the lean but did not significantly change in the obese. Lipid oversupply resulted in an increase $(P \leq 0.05)$ in methylation of the cytosine at position 14 (41,044 bp relative to the TSS) in both groups: lean from $95.2 \pm 0.4 \%$ to $96.5 \pm 0.5 \%$ and severely obese from $94.8 \pm 0.3 \%$ to $95.9 \pm 0.3 \%$ (Fig. 4 and Table 2). When absolute methylation percentage of the PPAR $\delta$ gene with lipid treatment was compared between lean and obese women, lipid oversupply resulted in a significantly lower $(P \leq 0.05)$ percentage of methylation among two cytosines at positions 6 (lean $6.4 \pm 0.7 \%$ vs. obese $4.3 \pm 0.4 \%$ ) and 7 (lean $9.7 \pm 1.3 \%$ vs. obese $6.3 \pm 0.4 \%$ ) in HSkMC from obese subjects (Fig. 4 and Table 2). There was a trend for the methylation of two cytosines at positions $4(P=0.08)$ and 5 $(P=0.06)(-78$ and -75 bp relative to the TSS $)$ to be lower in the severely obese compared with lean in the lipid-treated condition. With lipid treatment the change in methylation of the cytosine at position $6(-71 \mathrm{bp}$ from TSS) was positively related $(\mathrm{r}=0.64, P \leq 0.01)$ to the relative change in PPAR $\delta$ mRNA content (Fig. 5). Absolute methylation of the cytosine

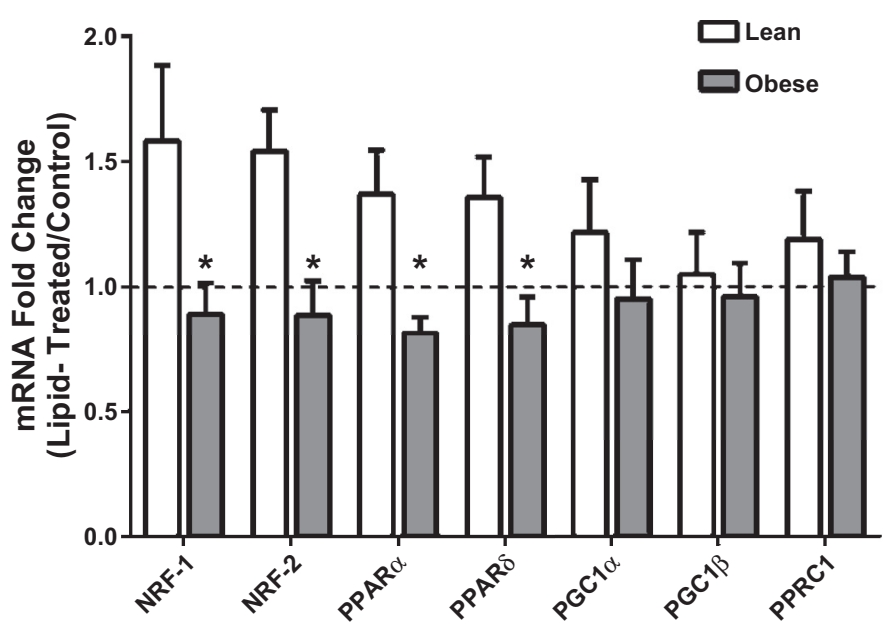

Fig. 1. Relative changes of peroxisome proliferator-activated receptor (PPAR) and nuclear respiratory factor (NRF) mRNA in response to lipid oversupply. Individual fold-changes in mRNA content in response to $48 \mathrm{~h} 250 \mu \mathrm{M}$ oleate-palmitate treatment in cultured myotubes [human skeletal muscle cells (HSkMC)] from lean (open bars) and severely obese (solid bars) women. Data are expressed as the fold-change (lipid-treated divided by control for each subject) (means $\pm \mathrm{SE}$ ). No change is a value of 1 , which is represented by the dashed line, with values $>1.0$ indicative of an increase in respective mRNA content with the lipid-treatment. *Significant difference $(P \leq 0.05)$ between lean and severely obese women. 
A

PPAR Regulated Genes

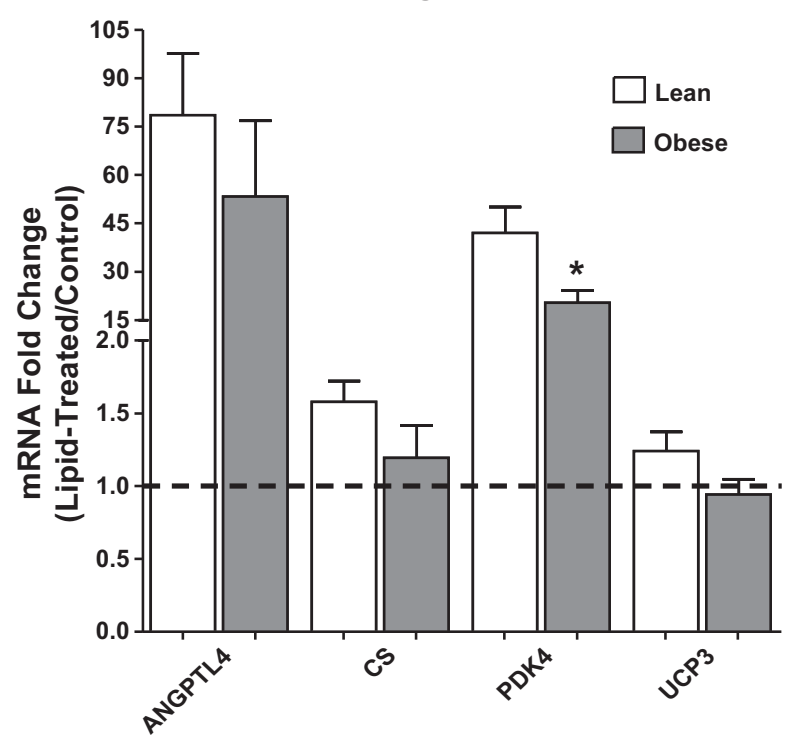

B

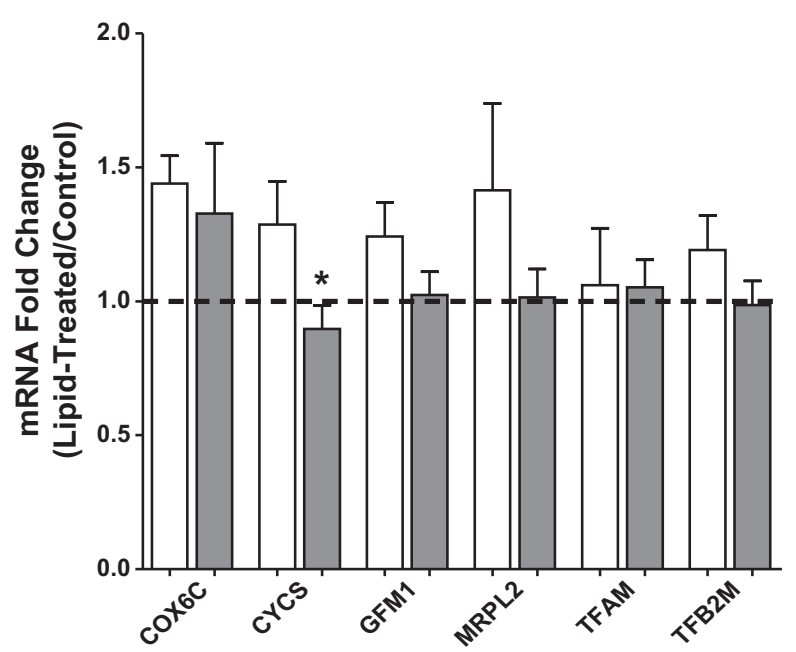

Fig. 2. Relative changes of PPAR- and NRF-regulated genes. Individual fold-changes in the mRNA content of PPAR $(A)$ and NRF-regulated genes $(B)$ in response to $48 \mathrm{~h} 250 \mu \mathrm{M}$ oleate-palmitate treatment in cultured myotubes (HSkMC) from lean (open bars) and severely obese (solid bars) women. Data are expressed as the fold-change (lipid-treated divided by baseline control for each subject) (means $\pm \mathrm{SE}$ ). No change is a value of 1 , which is represented by the dashed line, with values $>1.0$ indicative of an increase in respective mRNA content with the lipid treatment. *Significant difference $(P \leq 0.05)$ between lean and severely obese women.

at position 6 was positively related $(\mathrm{r}=0.53, P \leq 0.05)$ with $P P A R \delta$ mRNA content measured in the lipid-treated condition (Fig. 5). Methylation did not differ prior to lipid incubation (control condition) nor change significantly in any of the other genes examined.

Protein content. To assess the effects of changes in PDK4 mRNA levels on protein content, we measured protein levels by Western blot. In response to lipid exposure, PDK4 protein content increased $(P=0.01)$; however, there was no interaction effect $(P=0.83)$, nor were there any differences in PDK4 individual fold-changes between the lean and severely obese (lean $+11.2 \pm 6.7 \%$ vs. obese $+13.6 \pm 5.4 \%, P=0.78$ ). Additionally, there were no lipid-treatment or interaction effects for any of the other proteins assessed (PPAR $\alpha$, PPAR $\delta$, NRF-2, CYCS).

$F A O$. FAO was significantly depressed in the muscle cells from the severely obese women compared with lean women in both the baseline control and lipid-treated conditions (Fig. 6). While complete FAO rate significantly increased in response to $48 \mathrm{~h}$ lipid treatment in both groups, muscle cells from severely
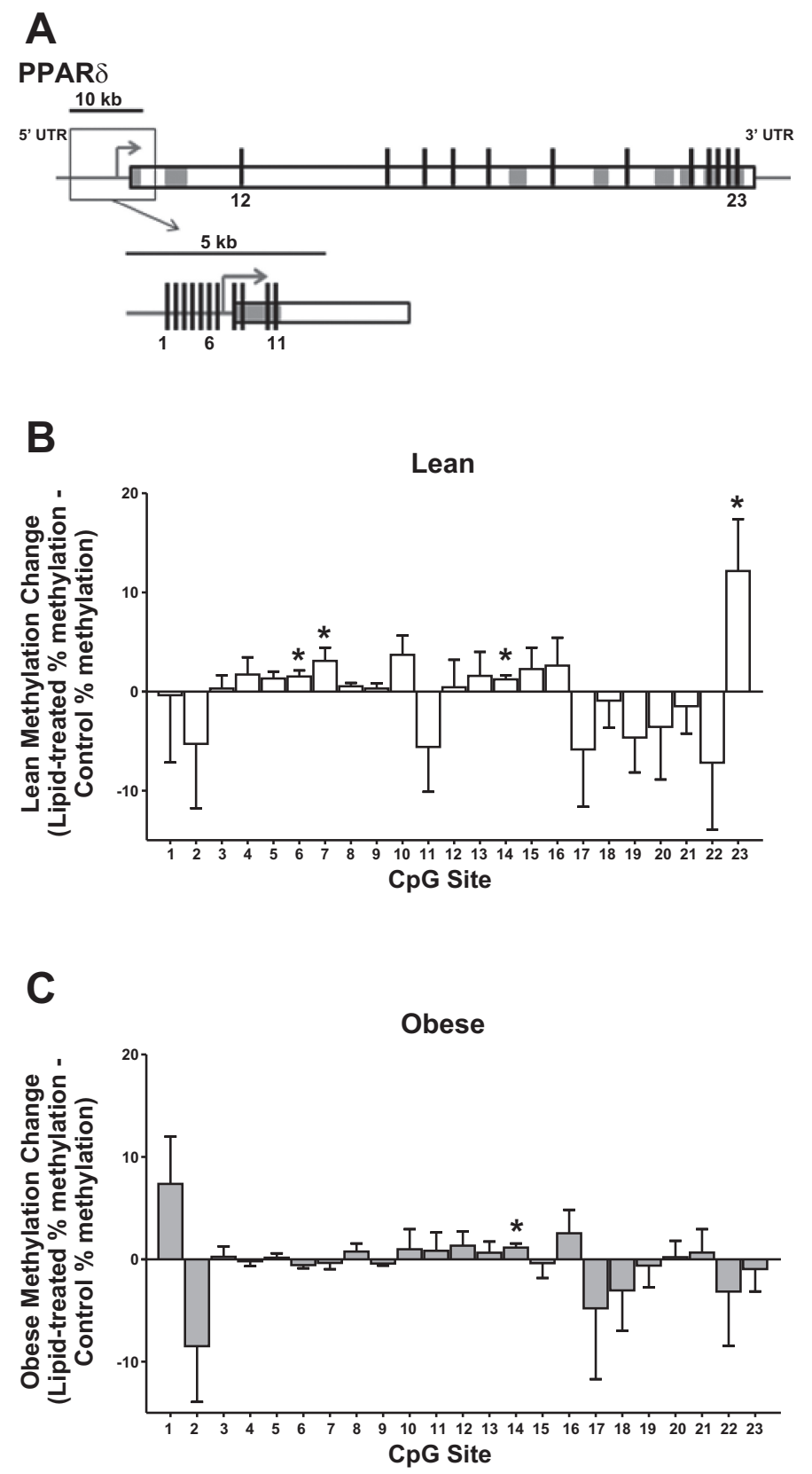

Fig. 3. PPAR $\delta$ gene methylation. Approximate location of the 23 measured $\mathrm{CpG}$ sites within the PPAR $\delta$ gene $(A)$. DNA methylation changes in response to lipid oversupply (methylation value in the lipid-treated condition minus the baseline control methylation value) at each of the 23 measured $\mathrm{CpG}$ sites among the lean $(B)$ and the severely obese women $(C)$. Transcription start site (TSS) is indicated by the arrow. * Significant difference $(P \leq 0.05)$ between the baseline control and lipid-treated condition. 
Table 2. Absolute percent methylation levels of individual cytosines within the PPAR gene

\begin{tabular}{|c|c|c|c|c|c|c|c|c|c|c|c|c|c|}
\hline \multirow[b]{2}{*}{ Cytosine Position \# } & \multirow[b]{2}{*}{ Distance from TSS } & \multicolumn{3}{|c|}{ Lean Control } & \multicolumn{3}{|c|}{ Lean Lipid-treated } & \multicolumn{3}{|c|}{ Obese Control } & \multicolumn{3}{|c|}{ Obese Lipid-treated } \\
\hline & & $n$ & Mean, \% & SE, \% & $n$ & Mean, \% & SE, $\%$ & $n$ & Mean, $\%$ & SE, $\%$ & $n$ & Mean, $\%$ & SE, $\%$ \\
\hline 1 & -468 & 9 & 60.5 & 2.3 & 9 & 60.1 & 6.4 & 9 & 58.0 & 3.1 & 9 & 62.6 & 3.9 \\
\hline 2 & -407 & 9 & 90.3 & 1.9 & 8 & 84.6 & 5.8 & 9 & 92.8 & 0.8 & 9 & 85.9 & 5.6 \\
\hline 3 & -212 & 9 & 4.9 & 0.6 & 6 & 4.6 & 0.6 & 9 & 5.4 & 0.7 & 9 & 5.7 & 0.8 \\
\hline 4 & -78 & 9 & 12.3 & 1.1 & 8 & 13.1 & 1.6 & 9 & 10.6 & 0.8 & 8 & 9.71\# & 0.6 \\
\hline 5 & -75 & 9 & 3.3 & 0.3 & 8 & 4.4 & 0.7 & 9 & $2.57 \#$ & 0.3 & 8 & 2.84\# & 0.3 \\
\hline 6 & -71 & 9 & 5.1 & 0.3 & 8 & 6.4 & 0.7 & 9 & 5.1 & 0.5 & 8 & $4.25^{*}$ & 0.4 \\
\hline 7 & -61 & 9 & 7.0 & 0.3 & 8 & 9.7 & 1.3 & 9 & 7.3 & 0.5 & 8 & $6.27 *$ & 0.4 \\
\hline 8 & 207 & 9 & 2.7 & 0.2 & 9 & 3.3 & 0.4 & 9 & 2.6 & 0.2 & 9 & 3.3 & 0.8 \\
\hline 9 & 350 & 9 & 4.0 & 0.3 & 9 & 4.3 & 0.5 & 9 & 3.8 & 0.1 & 8 & 3.4 & 0.2 \\
\hline 10 & 1199 & 9 & 6.5 & 2.4 & 8 & 10.7 & 3.7 & 7 & 3.4 & 0.4 & 7 & 3.8 & 1.6 \\
\hline 11 & 2507 & 9 & 79.6 & 1.7 & 9 & 74.0 & 4.5 & 9 & 74.12\# & 2.3 & 9 & 73.5 & 2.0 \\
\hline 12 & 17580 & 9 & 7.4 & 1.7 & 8 & 7.8 & 1.3 & 9 & 5.5 & 0.3 & 9 & 6.8 & 1.2 \\
\hline 13 & 38407 & 9 & 86.4 & 1.7 & 9 & 88.0 & 1.7 & 9 & 86.5 & 1.0 & 9 & 87.2 & 1.5 \\
\hline 14 & 41044 & 9 & 95.2 & 0.4 & 9 & 96.5 & 0.5 & 9 & 94.8 & 0.3 & 9 & 95.9 & 0.3 \\
\hline 15 & 43714 & 9 & 87.2 & 1.9 & 9 & 89.4 & 2.1 & 9 & 84.5 & 2.5 & 9 & 83.5 & 3.0 \\
\hline 16 & 50380 & 9 & 80.4 & 2.2 & 9 & 83.1 & 3.3 & 9 & 78.8 & 1.2 & 9 & 82.0 & 2.2 \\
\hline 17 & 59788 & 9 & 90.8 & 1.2 & 9 & 84.9 & 5.8 & 9 & 88.2 & 1.4 & 9 & 90.0 & 2.1 \\
\hline 18 & 76869 & 9 & 87.0 & 2.2 & 9 & 86.1 & 1.9 & 9 & 88.2 & 0.9 & 9 & 88.8 & 1.4 \\
\hline 19 & 81911 & 9 & 83.2 & 2.3 & 9 & 78.6 & 4.7 & 9 & 80.9 & 1.9 & 9 & 80.2 & 3.2 \\
\hline 20 & 81971 & 9 & 84.8 & 2.8 & 9 & 81.2 & 4.5 & 9 & 84.1 & 1.9 & 9 & 84.6 & 2.5 \\
\hline 21 & 82046 & 9 & 89.8 & 4.5 & 9 & 88.3 & 3.5 & 9 & 92.9 & 0.8 & 9 & 94.1 & 1.8 \\
\hline 22 & 82401 & 9 & 82.5 & 2.0 & 9 & 75.3 & 6.6 & 9 & 80.6 & 2.0 & 9 & 82.4 & 3.5 \\
\hline 23 & 84874 & 9 & 59.7 & 4.0 & 9 & 71.8 & 3.9 & 9 & 63.9 & 2.5 & 9 & 64.8 & 2.3 \\
\hline
\end{tabular}

PPAR, peroxisome proliferator-activated receptor; TSS, transcription start site. *Lean vs. obese $P<0.05$; \#Lean vs. obese $P<0.10$.

obese women had a smaller elevation (1.59 \pm 0.04 -fold) compared with the lean $(1.94 \pm 0.04$-fold, $P<0.05)$ indicating a decrement in metabolic flexibility in response to fatty acid exposure with severe obesity.

\section{DISCUSSION}

The current study examined if the expression of genes linked with FAO differ in response to lipid exposure in a manner indicative of a lack of metabolic flexibility with severe obesity and if these differences could be explained by disparate DNA methylation signatures. The main findings are: 1) increases in the expression of transcriptional regulators of FAO were depressed in HSkMC from severely obese individuals in response to lipid oversupply, and 2) PPAR $\delta$ expression in response to lipid may be influenced by transient changes in $\mathrm{CpG}$ methylation with the methylation pattern of the PPAR $\delta$ gene differing with severe obesity.

The PPARs and NRFs activate gene expression programs critical to FAO $(18,19,22,23)$. Of the three PPAR subtypes, PPAR $\alpha$ is expressed predominately in tissues that are characterized by high rates of FAO (i.e., liver, heart, muscle, kidney) and mediates the lipid-induced activation of genes involved in FAO. The importance of this transcription factor is supported by the observation that PPAR $\alpha$ knockout mice exhibit a dramatic inhibition of fatty acid uptake and oxidation, abnormal accumulation of lipids in oxidative tissues, and a failure to induce $\beta$-oxidation in response to physiological challenges such as a high-fat diet (24). PPAR $\delta$ is expressed in skeletal

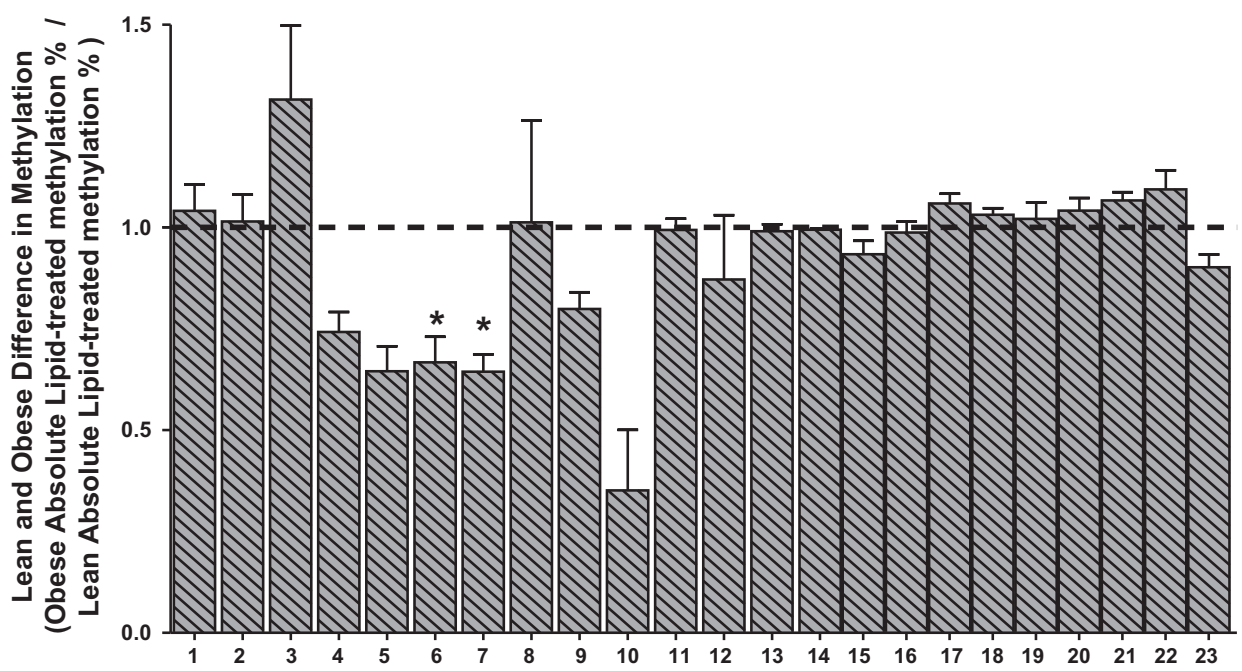

Fig. 4. PPAR $\delta$ gene methylation in the lipidtreated condition. Differences in the methylation status of 23 cytosines within the PPAR gene in the lipid-treated condition between lean and severely obese women. Data are presented as absolute lipid-treated methylation values for the severely obese relative to absolute lipid-treated methylation values for the lean (the obese lipid-treated methylation value divided by the lean lipid-treated methylation) (means $\pm \mathrm{SE}$ ). No differences in the methylation of cytosines in the lipid-treated condition between lean and obese is a value of 1 , which is represented by the dashed line, with values $<1.0$ indicative of lower methylation levels among the severely obese compared with the lean. *Significant difference $(P \leq 0.05)$ between lean and severely obese women. 
A

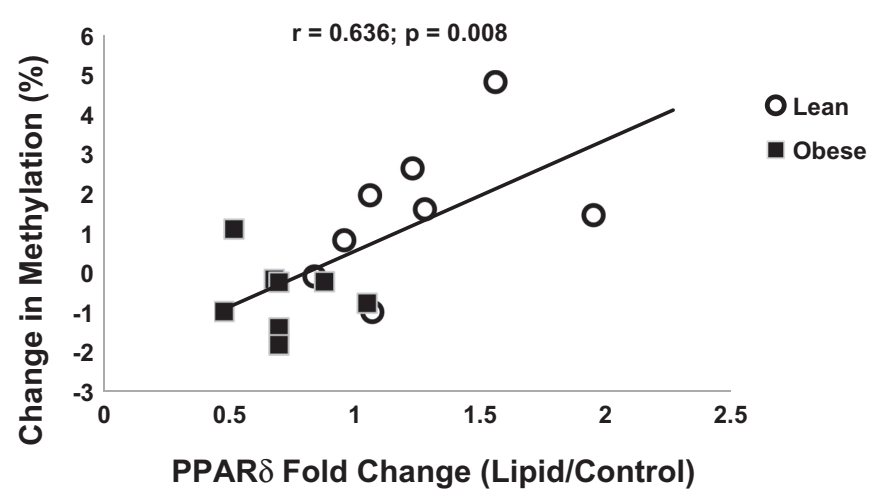

B

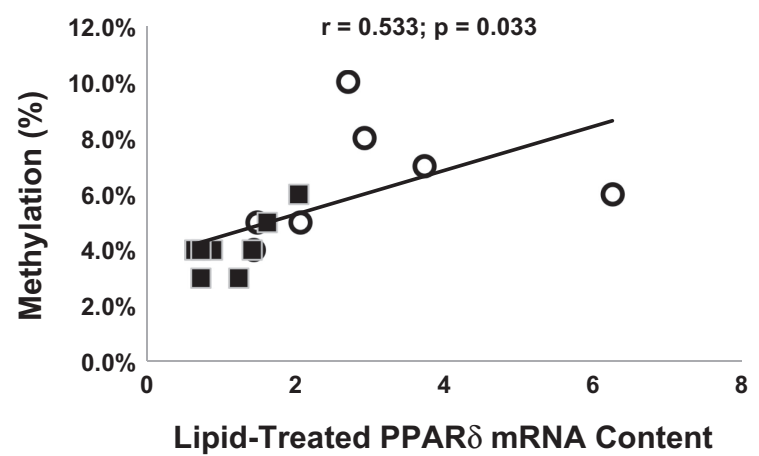

Fig. 5. The association of PPAR $\delta$ methylation and PPAR $\delta$ mRNA content. The change in methylation in response to lipid oversupply (methylation value in the lipid-treated condition minus the baseline control methylation value) of the cytosine at position 6 was positively related to the individual relative change in PPAR $\delta$ mRNA content (lipid-treated divided by baseline control for each subject) $(A)$. Absolute methylation of the cytosine at position 6 in the lipid-treated condition was positively related to PPAR $\delta$ mRNA content in the lipid-treated condition $(B)$.

muscle and plays role similar to PPAR $\alpha(19,23,29)$. The NRFs are required for the expression of the respiratory apparatus in mammalian cells and vital for mitochondrial biogenesis/maintenance as indicated by early mortality of $N R F-1$-null embryos (18).

In the current study, transcriptional regulators $P P A R \alpha$, PPARS, NRF-1, and NRF-2 exhibited similar patterns of increasing mRNA content with lipid incubation in the skeletal muscle of lean but not severely obese women (Fig. 1); this pattern of change approximated that for the functional measure of FAO, which was also repressed with severe obesity (Fig. 6). In the present study, we cannot discount the possibility that the differential gene expression patterns between lean and obese individuals may have been influenced by the experimental design, i.e., that similar mRNA responses could have occurred at earlier or later time points during lipid incubation; also, differences may have been more pronounced if different sampling times/lipid treatments had been examined. It is well known that different fatty acids impart specific and unique effects, or even opposing actions, on cellular functions (30); we chose to utilize the oleate-palmitate mixture as it more closely mimics physiological conditions (30). Regardless of these potential limitations, the present data still provide the finding that there is a coordinated lipid-induced activation of genes linked with FAO in the skeletal muscle of lean individuals that is largely absent with severe obesity in response to a physiologically relevant concentration of lipid.

We hypothesized that epigenetic processes (e.g., DNA methylation) could provide a mechanism explaining, at least in part, the differential mRNA responses in the skeletal muscle of lean vs. severely obese women. However, of the genes examined, only PPAR $\delta$ changed in a manner supportive of this hypothesis, suggesting that acute changes in methylation may not be a predominant mechanism for controlling fatty acid-induced changes in mRNA. With lipid oversupply there were significant increases in methylation in a cluster of cytosines proximal to the TSS in the lean but not severely obese women (Fig. 3), which may coincide with the cis-acting regulatory domain for $P P A R \delta$. Of the cytosines exhibiting a differential methylation signature within the cluster, one $(-71 \mathrm{bp}$ from the TSS at position 6) was highly related to PPAR $\delta$ mRNA content (Fig. $5)$. These data extend findings from others showing that individuals at a greater risk of developing metabolic disease exhibit a lower sensitivity to environmental challenges (i.e., high-fat feeding) in terms of the ability to regulate changes in DNA methylation (9).

DNA methylation is generally accepted to regulate gene transcription by directly impeding the binding of transcription factors to their target sites and through the recruitment of methyl-binding proteins (21). However, our findings indicate a positive relationship between the extent of PPAR $\delta$ promoter methylation and PPAR $\delta$ mRNA content in response to lipid (Fig. 5). In agreement, Barres et al. (2) identified a subset of genes in human skeletal muscle with positive relationships between gene expression and promoter methylation and suggested that DNA methylation at a transcriptional repressor binding site could subsequently induce gene expression. Pipaon et al. (26) found that increased methylation of $p 73$, a gene related to the p53 tumor suppressor protein, blocked the binding of the zinc finger transcription factor repressor protein ZEB in human fibroblasts, in turn promoting the expression of $p 73$ (26), and Ando et al. (1) found that demethylation of a repressor binding site elicited a concomitant decrease in gene expression (1). Although correlative at this point, our findings suggest that a potential mechanism regulating the lipid-induced increase in PPAR $\delta$ gene expression could be the blockage of a repressive factor from binding to the PPAR $\delta$ promoter by DNA

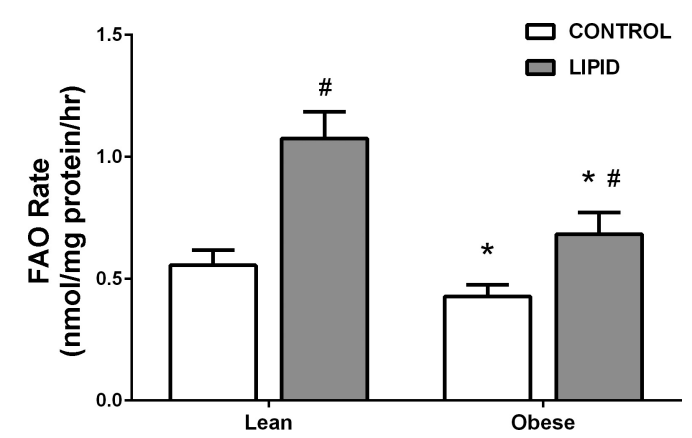

Fig. 6. Fatty acid oxidation (FAO) before and after $48 \mathrm{~h}$ of lipid exposure. FAO before and after $48 \mathrm{~h}$ oleate-palmitate treatment (lipids) in cultured myotubes (HSkMC) from lean and severely obese women. ${ }^{*}$ Significant difference $(P \leq$ $0.05)$ between lean and severely obese women for that condition. \#Significant increase $(P \leq 0.05)$ with lipid incubation. 
methylation. This observation raises the question of how methylation may be targeted specifically to the PPAR $\delta$ promoter in response to lipid exposure in lean subjects, or how the loss of methylation may be mediated in severely obese subjects. It has been proposed that site-specific methylation is itself regulated by DNA-binding transcription factors through several possible mechanisms that can both induce and prevent cytosine methylation, including the recruitment of DNA methyltransferases (DNMTs) to induce methylation, the steric blocking of DNMT to prevent methylation, or recruitment proteins such as teneleven-translocation that can oxidize 5-methylcytosine and thereby reverse DNA methylation $(5,25)$. Regardless of the specific mechanism involved, a significant and novel finding presented here is that this process may be regulated differently in lean vs. severely obese women, resulting in the disparate response to lipid exposure. However, this type of regulation may be specific to lipid-mediated responses as PPAR $\delta$ gene expression was repressed with obesity in the control (no lipid) condition (RESULTS) and FAO was lower prior to lipid exposure despite no initial differences in methylation status (Fig. 6).

In support of the physiological relevance of the changes in methylation frequencies observed, others have observed methylation changes of similar magnitude in parallel with altered expression levels mediated by diet and obesity $(2,3,16)$. Additionally, the magnitude of the differences in methylation frequency response at the specific cytosines in the PPAR $\delta$ $5^{\prime}$-flanking region between lean and obese subjects (Figs. 3 and 4) is commensurate with differences between differentially methylated genes associated with obesity observed by others $(2,6,20,27)$. A survey of these studies shows that commonly applied thresholds for functionally relevant changes in methylation are 10-20\%, while the increased methylation frequency at specific $P P A R \delta$ cytosines induced by lipid was $30-50 \%$, and the difference in this response between lean and obese was $\sim 40 \%$ (Figs. 3 and 4 ). Studies by others have also shown a similar level of correlation between methylation frequency and transcript level at $P G C-1 \alpha$ (3) as we observed for PPAR $\delta$ (Fig. 5). While the accordance of our findings with the body of published observations from similar studies supports their relevance, establishing an direct causal relationship between changes in methylation frequency and gene expression levels remains a challenge not just in the resolution of metabolically sensitive gene regulation in skeletal muscle, but in establishing the broader role of DNA methylation as an active gene regulatory mechanism. Thus, evidence in support of the causality between DNA methylation and gene expression must currently rely heavily on the preponderance of studies that show a correlation between site-specific methylation and gene expression levels as is presented here.

The differences in the response of muscle cells from lean and severely obese women to lipid treatment raises an important question regarding the underlying mechanisms that mediate this distinction. An important aspect of this question is the fact that the primary muscle cell cultures displayed these differences under identical conditions, indicating that the differences were cell-autonomous and not dependent on continued exposure to distinct environments. One possibility is that there are genetic differences between subject groups that correlate with severe obesity and affect the response to lipid in muscle cells, although the emergence of a genetic correlation with this number of subjects is highly improbable. An alterna- tive and more likely possibility is that a distinction in the environments to which the muscle cells were exposed in the lean and severely obese women resulted in a stable epigenetic reprogramming of key genes that led to the differential response to lipid. Consistent with this, Barres et al. (2) have suggested that adiposity, insulin levels, blood lipids, and inflammatory markers may have promoter-specific effects on DNA methylation. In the current study, there were no differences in the baseline methylation of the genes studied, indicating that a global alteration of DNA methylation mechanisms is unlikely. Instead, differential gene-specific DNA methylation and expression profile between lean and severely obese individuals in response to lipid oversupply suggests a more specific effect on lipid-mediated pathways, which could also involve other epigenetic modifications, such as histone acetylation or methylation. Resolving these questions will be a significant challenge but will ultimately clarify mechanisms underlying the correlation between obesity and metabolic derangement.

Functional determinations related to the metabolic profile of the myotubes such as protein expression (RESULTS) and FAO (Fig. 6) were also obtained as indexes of metabolic flexibility. The current data support our previous findings (8) albeit under slightly different conditions (i.e., $48 \mathrm{~h}$ vs. $24 \mathrm{~h}$ lipid incubation, $250 \mu \mathrm{M}$ vs. $100 \mu \mathrm{M}$ oleate-palmitate) of a dampened capacity to increase lipid oxidation upon lipid exposure with severe obesity. Although some aspects of gene expression and methylation status also differed in a manner reflective of this lack of responsiveness (Figs. 1-4), similar patterns were not evident in protein content, with only PDK4 content increasing and to a similar extent regardless of obesity status (RESULTS). Together, these data suggest that a more prolonged and/or extensive lipid stimulus could be needed for differences in mRNA content to become manifest in protein expression. In addition, the PPAR/ NRF axis, at least in relation to acute changes in protein content, may not be extensively involved in regulating FAO under the experimental conditions utilized. However, these hypotheses need to be validated by future studies.

In summary, primary HSkMC cultures were utilized to study responses to a lipid stimulus in the skeletal muscle of lean and severely obese women. Our findings indicate a coordinated lipid-induced activation of genes linked with FAO among lean individuals in response to lipid oversupply that is largely absent with severe obesity. In HSkMC from severely obese women, PPAR $\delta$ displayed differential methylation patterns in the promoter region compared with cells derived from lean subjects; transient changes in DNA methylation patterns may thus play a role in the transcriptional regulation of PPAR $\delta$ by lipid exposure. The cell-autonomous differences in the response of this regulatory mechanism between lean and obese subjects suggest that an epigenetic process sensitive to an obesogenic environment may underlie the metabolic changes in skeletal muscle that accompany obesity.

\section{ACKNOWLEDGMENTS}

The authors thank Amanda K. Staples and Lauren M. Cox for technical assistance in data collection.

\section{GRANTS}

Funding provided by National Institutes of Health Grants DK-056112 (J. A. Houmard) and 2P20GM-103436-14 (J. M. Maples). 


\section{DISCLOSURES}

No conflicts of interest, financial or otherwise, are declared by the author(s).

\section{AUTHOR CONTRIBUTIONS}

Author contributions: J.M.M. and J.A.H. conception and design of research; J.M.M., K.Z., N.R., M.J.H., and T.M.W. performed experiments; J.M.M., J.J.B., B.M.S., K.Z., N.R., M.J.H., and J.A.H. analyzed data; J.M.M., J.J.B., B.M.S., C.A.W., K.Z., N.R., M.J.H., and J.A.H. interpreted results of experiments; J.M.M. and K.Z. prepared figures; J.M.M., B.M.S., and J.A.H. drafted manuscript; J.M.M., J.J.B., B.M.S., C.A.W., K.Z., T.M.W., and J.A.H. edited and revised manuscript; J.M.M., J.J.B., B.M.S., C.A.W., K.Z., N.R., M.J.H., T.M.W., and J.A.H. approved final version of manuscript.

\section{REFERENCES}

1. Ando T, Nishimura M, Oka Y. Decitabine (5-Aza-2'-deoxycytidine) decreased DNA methylation and expression of MDR-1 gene in K562/ ADM cells. Leukemia 14: 1915-1920, 2000.

2. Barres R, Kirchner H, Rasmussen M, Yan J, Kantor FR, Krook A, Näslund E, Zierath JR. Weight loss after gastric bypass surgery in human obesity remodels promoter methylation. Cell Rep 3: 1020-1027, 2013.

3. Barres R, Osler ME, Yan J, Rune A, Fritz T, Caidahl K, Krook A, Zierath JR. Non-CpG methylation of the PGC-1alpha promoter through DNMT3B controls mitochondrial density. Cell Metab 10: 189-198, 2009.

4. Berggren JR, Tanner CJ, Houmard JA. Primary cell cultures in the study of human muscle metabolism. Exerc Sport Sci Rev 35: 56-61, 2007.

5. Blattler A, Farnham PJ. Cross-talk between site-specific transcription factors and DNA methylation states. J Biol Chem 288: 34287-34294, 2013.

6. Borengasser SJ, Zhong Y, Kang P, Lindsey F, Ronis MJ, Badger TM, Gomez-Acevedo H, Shankar K. Maternal obesity enhances white adipose tissue differentiation and alters genome-scale DNA methylation in male rat offspring. Endocrinology 154: 4113-4125, 2013.

7. Boyle KE, Canham JP, Consitt LA, Zheng D, Koves TR, Gavin TP, Holbert D, Neufer PD, Ilkayeva O, Muoio DM, Houmard JA. A high-fat diet elicits differential responses in genes coordinating oxidative metabolism in skeletal muscle of lean and obese individuals. J Clin Endocrinol Metab 96: 775-781, 2011.

8. Boyle KE, Zheng D, Anderson EJ, Neufer PD, Houmard JA. Mitochondrial lipid oxidation is impaired in cultured myotubes from obese humans. Int J Obes 25: 201, 2011.

9. Brøns C, Jacobsen S, Nilsson E, Rönn T, Jensen CB, Storgaard H, Poulsen P, Groop L, Ling C, Astrup A, Vaag A. Deoxyribonucleic acid methylation and gene expression of PPARGC1A in human muscle is influenced by high-fat overfeeding in a birth-weight-dependent manner. $J$ Clin Endocrinol Metab 95: 3048-3056, 2010.

10. Cameron-Smith D, Burke LM, Angus DJ, Tunstall RJ, Cox GR, Bonen A, Hawley JA, Hargreaves M. A short-term, high-fat diet upregulates lipid metabolism and gene expression in human skeletal muscle. Am J Clin Nutr 77: 313-318, 2003.

11. Crunkhorn S, Dearie F, Mantzoros C, Gami H, da Silva WS, Espinoza D, Faucette R, Barry K, Bianco AC, Patti ME. Peroxisome proliferator activator receptor gamma coactivator-1 expression is reduced in obesity: potential pathogenic role of saturated fatty acids and p38 mitogen-activated protein kinase activation. J Biol Chem 282: 15439-15450, 2007.

12. Evans WJ, Phinney SD, Young VR. Suction applied to a muscle biopsy maximizes sample size. Med Sci Sports Exerc 14: 101-102, 1982.
13. Hancock CR, Han DH, Chen M, Terada S, Yasuda T, Wright DC, Holloszy JO. High-fat diets cause insulin resistance despite an increase in muscle mitochondria. Proc Natl Acad Sci USA 105: 7815-7820, 2008.

14. Hock MB, Kralli A. Transcriptional control of mitochondrial biogenesis and function. Annu Rev Physiol 71: 177-203, 2009.

15. Huffman KM, Hawk VH, Henes ST, Ocampo CI, Orenduff MC, Slentz CA, Johnson JL, Houmard JA, Samsa GP, Kraus WE, Bales CW. Exercise effects on lipids in persons with varying dietary patternsdoes diet matter if they exercise? Responses in studies of a targeted risk reduction intervention through defined exercise I. Am Heart J 164: 117-124, 2012.

16. Jacobsen SC, Brons C, Bork-Jensen J, Ribel-Madsen R, Yang B, Lara E, Hall E, Calvanese V, Nilsson E, Jorgensen SW, Mandrup S, Ling C, Fernandez AF, Fraga MF, Poulsen P, Vaag A. Effects of short-term high-fat overfeeding on genome-wide DNA methylation in the skeletal muscle of healthy young men. Diabetologia 55: 3341-3349, 2012.

17. Kelley DE, Mandarino LJ. Fuel selection in human skeletal muscle in insulin resistance: a reexamination. Diabetes 49: 677-683, 2000.

18. Kelly DP, Scarpulla RC. Transcriptional regulatory circuits controlling mitochondrial biogenesis and function. Genes Dev 18: 357-368, 2004.

19. Lee CH, Olson P, Evans RM. Minireview: lipid metabolism, metabolic diseases, and peroxisome proliferator-activated receptors. Endocrinology 144: 2201-2207, 2003.

20. Milagro FI, Campion J, Cordero P, Goyenechea E, Gomez-Uriz AM, Abete I, Zulet MA, Martinez JA. A dual epigenomic approach for the search of obesity biomarkers: DNA methylation in relation to diet-induced weight loss. FASEB J 25: 1378-1389, 2011.

21. Miranda TB, Jones PA. DNA methylation: the nuts and bolts of repression. J Cell Physiol 213: 384-390, 2007.

22. Muoio DM, Koves TR. Skeletal muscle adaptation to fatty acid depends on coordinated actions of the PPARs and PGC1 alpha: implications for metabolic disease. Appl Physiol Nutr Metab 32: 874-883, 2007.

23. Muoio DM, MacLean PS, Lang DB, Li S, Houmard JA, Way JM, Winegar DA, Corton JC, Dohm GL, Kraus WE. Fatty acid homeostasis and induction of lipid regulatory genes in skeletal muscles of peroxisome proliferator-activated receptor (PPAR) alpha knock-out mice. Evidence for compensatory regulation by PPAR delta. J Biol Chem 277: 2608926097, 2002.

24. Muoio DM, Way JM, Tanner CJ, Winegar DA, Kliewer SA, Houmard JA, Kraus WE, Dohm GL. Peroxisome proliferator-activated receptoralpha regulates fatty acid utilization in primary human skeletal muscle cells. Diabetes 51: 901-909, 2002.

25. Pastor WA, Aravind L, Rao A. TETonic shift: biological roles of TET proteins in DNA demethylation and transcription. Nat Rev Mol Cell Biol 14: 341-356, 2013.

26. Pipaon C, Real PJ, Fernandez-Luna JL. Defective binding of transcriptional repressor ZEB via DNA methylation contributes to increased constitutive levels of p73 in Fanconi anemia cells. FEBS Lett 579: 4610-4614, 2005.

27. Rastogi D, Suzuki M, Greally JM. Differential epigenome-wide DNA methylation patterns in childhood obesity-associated asthma. Sci Rep 3: 2164, 2013.

28. Sandoval J, Heyn H, Moran S, Serra-Musach J, Pujana MA, Bibikova M, Esteller M. Validation of a DNA methylation microarray for 450,000 CpG sites in the human genome. Epigenetics 6: 692-702, 2011.

29. Wagner KD, Wagner N. Peroxisome proliferator-activated receptor $\beta / \delta$ $(\mathrm{PPAR} \beta / \delta)$ acts as regulator of metabolism linked to multiple cellular functions. Pharmacol Ther 125: 423-435, 2010.

30. Watt MJ, Hoy AJ, Muoio DM, Coleman RA. Distinct roles of specific fatty acids in cellular processes: implications for interpreting and reporting experiments. Am J Physiol Endocrinol Metab 302: E1-E3, 2012. 